\title{
Two-frequency forcing of droplet rebounds on a liquid bath
}

\author{
Naresh Sampara and Tristan Gilet ${ }^{1, *}$ \\ ${ }^{1}$ Microfluidics Lab, \\ Department of Aerospace and Mechanics, \\ University of Liège, B-4000 Liège, Belgium
}

\begin{abstract}
Droplets can bounce indefinitely on a liquid bath vertically vibrated in a sinusoidal fashion. We here present experimental results that extend this observation to forcing signals composed of a combination of two commensurable frequencies. The Faraday and Goodridge thresholds are characterized. Then a number of vertical bouncing modes are reported, including walkers. The vertical motion can become chaotic, in which case the horizontal motion is an alternation of walk and stop.
\end{abstract}

PACS numbers: 47.55.D-, 47.20.Ma, 47.35.Pq

\section{INTRODUCTION}

One of the hidden beauties of nature is that droplets smoothly landing on a liquid bath levitate for a few hundredths of seconds before they merge with the bath. Indeed, coalescence occurs only when the thin air layer between the droplet and the bath has drained out [1]. At non-zero impact velocity, the droplet may bounce a few times onto the bath surface, provided that the collision time is less than the drainage time [2]. Permanent levitation can be achieved by vertically vibrating the bath container with sufficient amplitude; the droplet then bounces periodically and the intervening air layer is replenished at each take-off $[3,4]$.

The rebound of droplets on a vibrated liquid bath has attracted much attention in the last decade, owing to its reminiscence of the wave-particle duality $[5,6]$. Indeed, every droplet impact on the vibrated interface generates standing Faraday waves [7]. As the droplet subsequently impacts on this wavy surface, it gains horizontal momentum and starts "walking" at the surface of the bath $[8,9]$. This coupled system of a droplet and a wave has shown several behaviors analog to quantum particles, including diffraction, tunneling, quantized orbits and wave-like statistics [10-14]. In addition, the vertical dynamics of bouncing droplets exhibits some features of low-dimensional chaotic oscillators $[15,16]$. Several previous works have characterized the modes of rebound of a droplet on a liquid substrate vibrated sinusoidally at a given frequency $f[9,17-20]$. This frequency corresponds to a given excited wavelength according to the dispersion relation of Faraday waves.

In this work, we characterize experimentally the vertical dynamics of a bouncing droplet on a bath vibrated simultaneously at two frequencies. We observe several regimes (incl. coalescence, bouncing, ejection) as the amplitude of each frequency contribution is varied. The possible bouncing modes are then extensively reported. Our investigation aims at paving the way to an exploration of the horizontal dynamics of walkers in such conditions.

\footnotetext{
* Tristan.Gilet@ulg.ac.be; http://labos.ulg.ac.be/microfluidics
}

\section{EXPERIMENTAL SETUP}

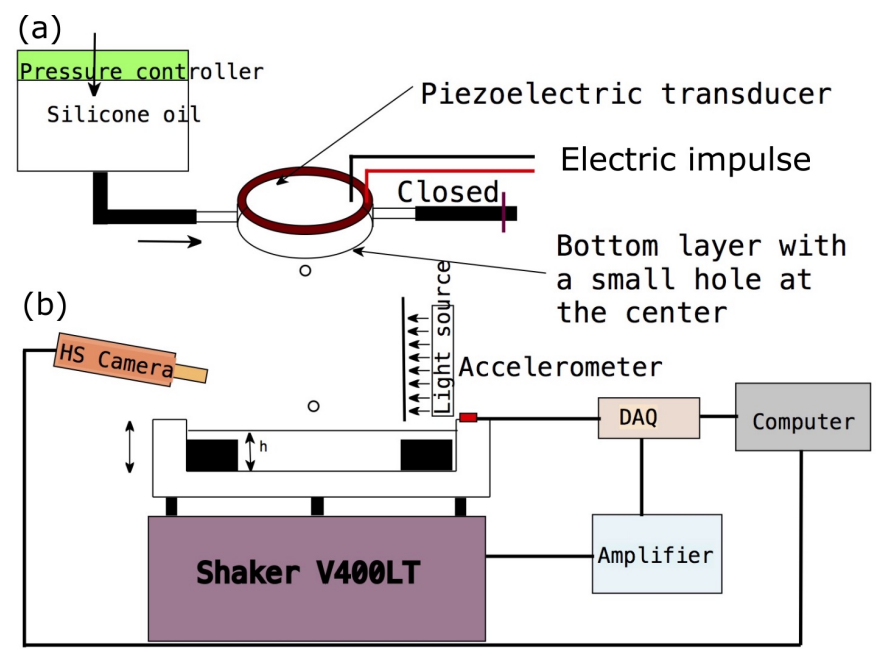

FIG. 1. Experimental setup: (a) droplet dispenser, and (b) vibrated bath. (a) A small container with a hole and a piezoelectric membrane is filled with silicone oil. An electric pulse creates a shock wave that ejects a droplet through the hole. (b) The droplet lands on a bath of silicone oil that is mounted on a shaker. The bath vibrates vertically according to a driving signal provided by a DAQ through an amplifier. The droplet is recorded with a high speed camera (back lighting).

A schematics of our experimental setup is shown in figure 1. It consists in a home-made droplet dispenser placed above a vertically vibrated container. The dispenser is a small circular cavity sandwiched between a piezoelectric transducer and a plastic sheet with a hole of diameter $800 \mu \mathrm{m}[21,22]$. It is filled with silicone oil (kinematic viscosity $\nu=20 \mathrm{~mm}^{2} / \mathrm{s}$, surface tension $\sigma=20.9 \mathrm{mN} \cdot \mathrm{m}^{-1}$ and density $\rho=956 \mathrm{~kg} \cdot \mathrm{m}^{-3}$ ) at a controlled pressure that ensures that neither can air bubbles enter the cavity, nor can the liquid leak out by itself. A short electric pulse (1 to $6 \mathrm{~ms}$ ) applied to the piezoelectric generates a shock wave in the container that expels a droplet of reproducible size through the hole. The droplet radius $R$ depends on the inlet pressure, the hole diameter and the applied voltage. It is here fixed to 
$R=375( \pm 25) \mu \mathrm{m}$. The droplet then lands and bounces on the underlying vibrated bath. Its motion is recorded optically with a high speed camera (Phantom MIRO110, 1600 frames per second) and some back lighting.

The circular container of inner diameter $280 \mathrm{~mm}$ and depth $10 \mathrm{~mm}$ is tightly fixed on an electromagnetic shaker (V400LT, Data Physics) connected to an amplifier (DSA5-2k, Data Physics). The container is filled with $5.5 \mathrm{~mm}$ of the same silicone oil as the droplet. The vibrating meniscus at the contact line generates parasitic gravity-capillary waves. These waves are immediately damped thanks to a beach structure of height $4.5 \mathrm{~mm}$ attached at the boundary of the container. Two accelerometers (PCB, 352C65) of sensitivity $\simeq 100 \mathrm{mV} / g$ ( $g$ is acceleration of gravity) are mounted at diametrically opposite positions on top of the container to measure the homogeneity of the vibration. A data acquisition device (DAQ - National instruments USB 6212, interfaced with Labview) records accelerometer measurements and generates the forcing signal accordingly. A feedback loop (timescale $1 \mathrm{~s}$ ) from the accelerometers to the gain of the amplifier maintains a vibration of constant amplitude.

The container is vibrated according to a forcing signal composed exclusively of the fourth and fifth harmonics of a fundamental frequency $f=16 \mathrm{~Hz}$ (pe$\operatorname{riod} T=62.5 \mathrm{~ms}$ ). These harmonics correspond to $4 f=64 \mathrm{~Hz}$ and $5 f=80 \mathrm{~Hz}$. Most previous studies on walkers have concentrated on a forcing frequency of $80 \mathrm{~Hz}$ and a kinematic viscosity of $20 \mathrm{~mm}^{2} / \mathrm{s}$, so we also consider $80 \mathrm{~Hz}$ as the $N$-th harmonic of our forcing signal. The second harmonic has been chosen to also belong to the walking window $[60,90] \mathrm{Hz}[23,24]$. Our choice $N=5$ is the smallest integer that satisfies the constraint $80(N-1) / N>60$.

We consider the case of no phase shift between harmonics. The container elevation $z_{c}(t)$ then satisfies

$$
\ddot{z}_{c}=\sum_{m \in\{4,5\}} \gamma_{m} g \sin (2 \pi m f t)
$$

where $\gamma_{m}$ is the dimensionless acceleration amplitude of the $m$-th harmonic. Amplitudes $\gamma_{4}$ and $\gamma_{5}$ are varied extensively between 0 and 7 throughout this study, while none of the other harmonics is excited.

In addition to amplitudes $\gamma_{m}$, three other independent dimensionless numbers can be defined from the six variables $f, \rho, \nu, \sigma, R$ and $g$. These numbers are fixed in our experiments:

- The Bond number $B o=\frac{\rho g R^{2}}{\sigma} \simeq 0.063$ is the ratio between gravity and surface tension. As it is much smaller than unity, the droplet remains spherical in good approximation. This is still true when the acceleration felt by the droplet is augmented by the vibration (i.e. when $g$ is replaced by $\gamma_{m} g$ ), as long as $\gamma_{m}<16$.

- The dimensionless viscosity $\frac{\nu}{m f R^{2}} \gtrsim 1.78$ is the ratio between the bouncing period $1 /(m f)$ and the viscous time $R^{2} / \nu$. Its value larger than unity indicates that droplet deformations are damped between two successive impacts.

- The vibration number $\Omega_{m}=2 \pi m f \sqrt{\rho R^{3} / \sigma}$ indicates how far each harmonic is from the first resonance frequency of the droplet $\Omega_{r}=\sqrt{8}$. It has indeed been observed $[4,18,20,21,25]$ that bouncing thresholds are significantly higher when $\Omega \sim \Omega_{r}$. Moreover, the walking window is within $0.4<\Omega<1$ for the considered oil viscosity [24]. Here, $\Omega_{4}=0.62$ and $\Omega_{5}=0.78$, so both chosen harmonics are in the walking window, as wanted.

\section{REGIMES OF FARADAY WAVES}

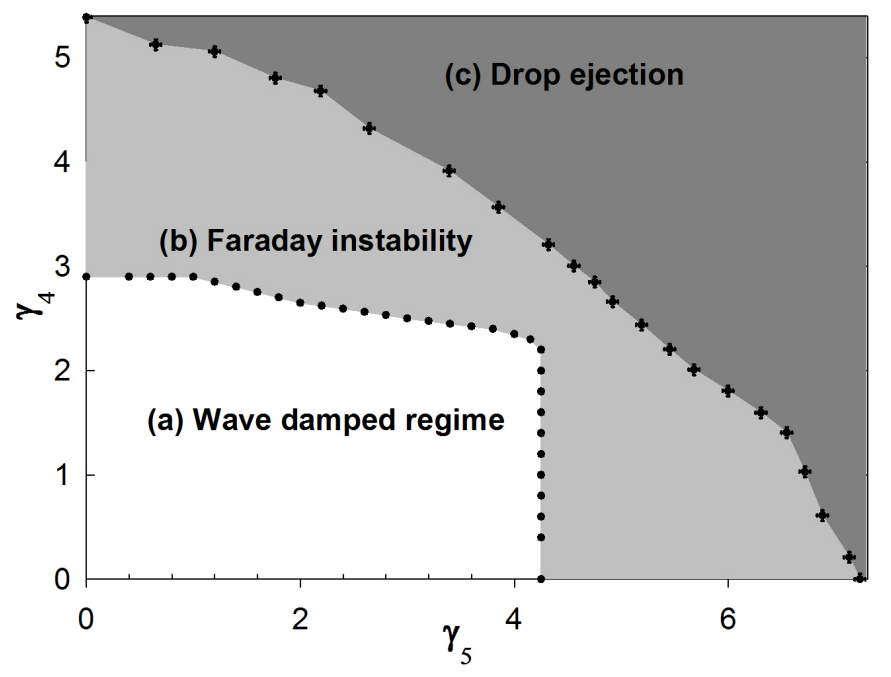

FIG. 2. Behavior of the vibrated bath as a function of forcing amplitudes $\left(\gamma_{4}, \gamma_{5}\right)$. Horizontal and vertical axes correspond to acceleration components $\gamma_{5}$ and $\gamma_{4}$ respectively (Eq. 1). Three different regimes are represented: damped waves (white), Faraday instability (gray) and droplet ejection (dark gray). The symbols correspond to experimental measurements. Errors on the Faraday instability threshold are estimated to be smaller than the symbol size.

As far as the bath only is concerned, three distinct regimes are observed as either or both accelerations $\gamma_{m}$ are increased: the damped waves, the Faraday instability, and the droplet ejection. They are reported in the phase diagram of figure 2 and detailed hereafter.

Each droplet impact (or any other perturbation of the bath surface) generates a gravity-capillary wave that travels radially outward. As soon as the bath is vibrated, this traveling wave excites Faraday waves on its way [9]. Faraday waves approximately satisfy the same dispersion relation as gravity-capillary waves, although their frequency is usually fixed to half the forcing frequency 
(sub-harmonic) [7]:

$$
\pi^{2}(m f)^{2}=\left(g k_{m}+\frac{\sigma}{\rho} k_{m}^{3}\right) \tanh \left(k_{m} h\right) .
$$

where $h$ is the liquid thickness and $k_{m}=2 \pi / \lambda_{m}$ is the Faraday wave number associated to the $m$-th harmonic.

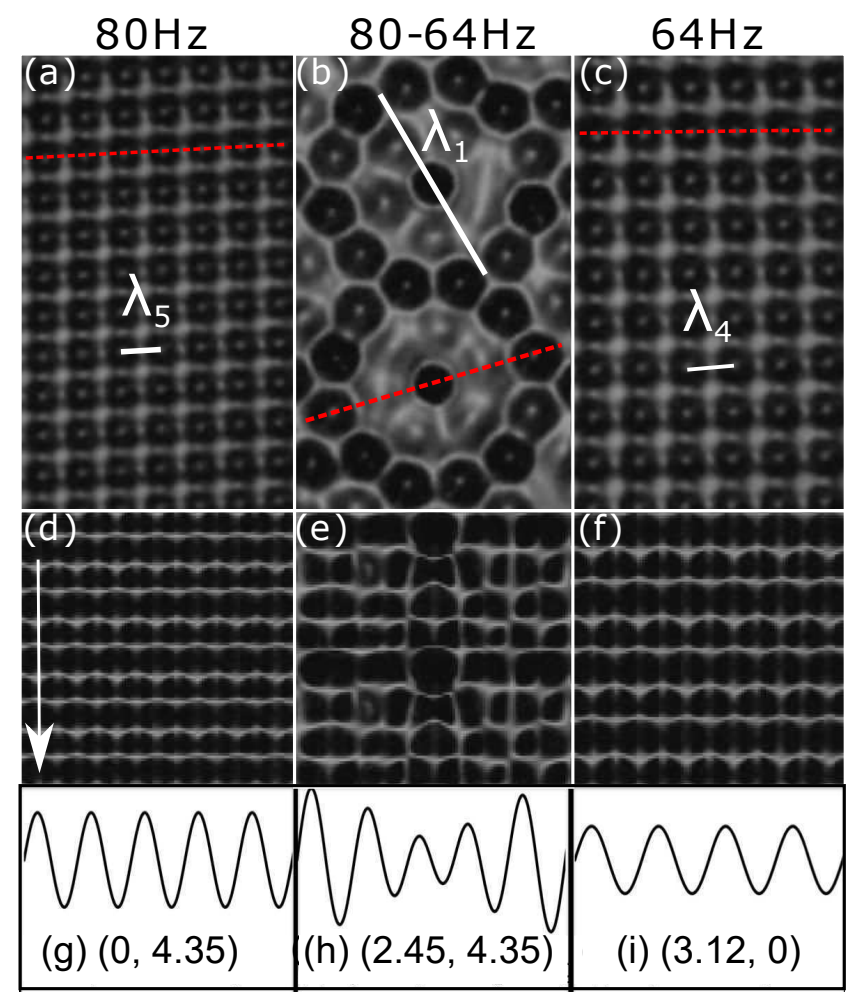

FIG. 3. (a)-(c) Top view of the standing wave pattern for various pairs $\left(\gamma_{4}, \gamma_{5}\right)$ : (a) $(0,4.35)$, (b) $(2.45,4.35)$ and (c) $(3.12,0)$. (d)-(f) Spatio-temporal diagrams obtained by vertically combining lines of pixels (dashed red) from (a-c) at different times. The time window (vertical axis) is $1 / 8 \mathrm{~s}$. (g)(i) Forcing signal from the shaker, corresponding to (a-d), (b,e) and (c,f) respectively.

At low forcing acceleration, Faraday waves are exponentially damped in time. Their amplitude also decreases with increasing distance from the source, as does the traveling wave. This temporal damping decreases with increasing forcing acceleration. The Faraday instability threshold is reached when the damping rate goes to zero. It is experimentally measured by direct observation of the light reflected from the liquid surface (with an accuracy of $0.1 \%$ ). The single-frequency thresholds (when only one harmonic is present) are $\gamma_{4 F}=2.92$ and $\gamma_{5 F}=4.25$ respectively. In the low viscosity regime $\left(\nu k^{2} \ll 2 \pi m f\right)$, they can be predicted by

$$
\gamma_{m F} \simeq 2 \alpha \frac{\nu k_{m}(2 \pi m f)}{g},
$$

where $\alpha$ is a dimensionless fitting parameter associated to viscous damping (cf. Appendix). Choosing $\alpha=1.58$ yields $\gamma_{4 F}=2.87$ and $\gamma_{5 F}=4.28$, in good agreement with experiments. The scaling law of Equation (3) can be retrieved by balancing the power dissipated by viscosity with the power transferred from the forcing. For a wave elevation of amplitude $h$, the corresponding vertical speed scales as $h m f$. The inertial force per unit of horizontal surface scales as the acceleration $\gamma g$ times the mass per unit surface $\rho h$ of the wave. The forcing power then scales as the force times the velocity, so as $(h m f) \cdot(\rho h) \cdot(\gamma g)$. The power dissipated by the flows in the bulk (again per unit surface) scales as $\rho \nu\left(h m f k_{m}\right)^{2} k_{m}^{-1}$, where the factor in brackets is proportional to the horizontal gradient of vertical velocity. Balancing both powers indeed yields $\gamma_{F} \sim\left(\nu k_{m} m f\right) / g$.

The regime of walking droplets is obtained at forcing acceleration slightly below the Faraday threshold; the Faraday waves generated by the droplet are then weakly damped. Above threshold, Faraday waves originate from any infinitesimal perturbation and they contaminate the whole surface of the bath (Fig. 3). Regular square patterns are observed for single-frequency forcing (Fig. 3a,c)[26]. At $\left(\gamma_{4}, \gamma_{5}\right)=(0,4.35)$ [resp. $\left.(3.12,0)\right]$, the measured wavelength is $\lambda_{5}=4.68 \pm 0.05 \mathrm{~mm}$ [resp. $\left.\lambda_{4}=5.55 \pm 0.05 \mathrm{~mm}\right]$, in good agreement with the theoretical value $\lambda_{5}=4.76 \mathrm{~mm}$ [resp. $\lambda_{4}=5.68 \mathrm{~mm}$ ]. The corresponding period $2 /(5 f)=(2 T / 5)=1 / 40 \mathrm{~s}$ [resp. $2 /(4 f)=T / 2=1 / 32 \mathrm{~s}]$ is checked on spatio-temporal diagrams (Fig. 3d,f).

For two-frequency forcing, the Faraday threshold is a hypersurface (here a curve) $\Gamma_{F}$ in the $\gamma_{m}$-space (Fig. 2). This curve intercepts the axes perpendicularly, which means that a slight presence of the second forcing frequency does not affect the instability as triggered by the first. More complex quasi patterns (octagonal and twelvefold) are produced when both accelerations are large $[27,28]$, e.g. at $\left(\gamma_{4}, \gamma_{5}\right)=(2.45,4.35)$ in Fig. 3b. Their spatial extension $\lambda_{1}=26.4 \mathrm{~mm}$ is very close to the theoretical wavelength $\lambda_{1}=27.3 \mathrm{~mm}$ associated to the fundamental frequency. The time evolution of these patterns, illustrated in the spatio-temporal diagram of Fig. 3e, is a complex mixture of both harmonics, of resulting period $1 / f=1 / 16 \mathrm{~s}$.

The amplitude of Faraday waves grows with increasing forcing acceleration. When the amplitude becomes comparable to the Faraday wavelength, the waves spike and break up into droplets (Fig. 4). This behavior, similar to the Rayleigh-Taylor instability, was reported and rationalized by Goodridge et al. [29-31] for the case of single-frequency forcing. Monodisperse aerosols for medical applications can be produced advantageously with this technique of droplet formation [32]. Here, the ejected droplets then bounce chaotically and they typically coalesce with the underlying liquid bath after a few impacts.

It is challenging to accurately determine the ejection acceleration threshold $\gamma_{d}$ experimentally, as there are very few droplet ejections for $\gamma \gtrsim \gamma_{d}$. We consider to be above the ejection threshold when there are more than 3 droplets ejected in $10 \mathrm{~s}$ over the whole bath sur- 


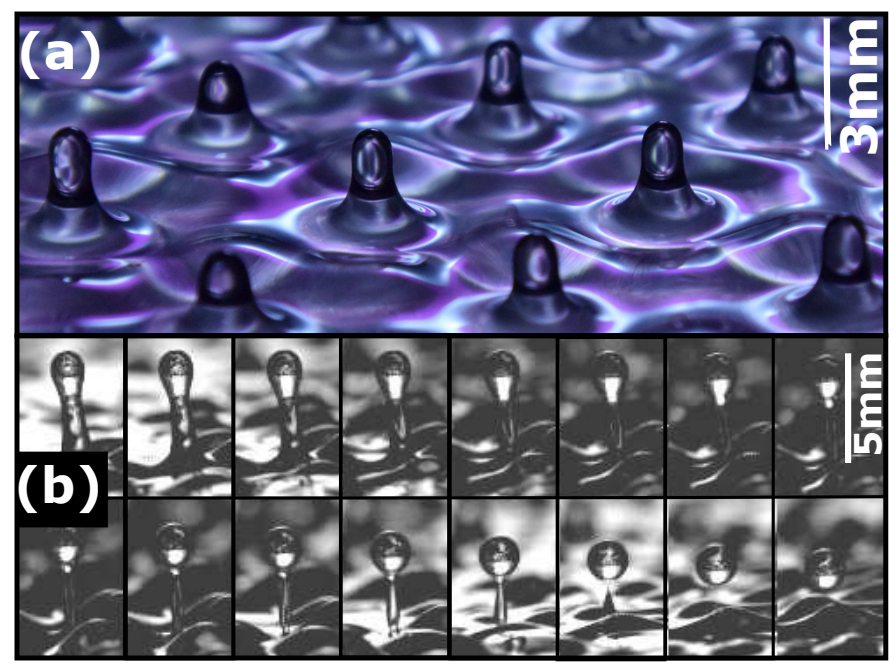

FIG. 4. (a) A side view of the extended Faraday waves at forcing acceleration $\left(\gamma_{4}, \gamma_{5}\right)=(2.54,4.47)$. (b) Time sequence of a droplet ejection event. Successive images are separated by $1 / 800 \mathrm{~s}$.

face. For single-frequency, the measured thresholds are $\gamma_{4 d}=(5.14 \pm 0.05)$ and $\gamma_{5 d}=(6.95 \pm 0.05)$. For twofrequency forcing, the ejection threshold is again a hypersurface $\Gamma_{d}$ in the $\gamma_{m}$-space (Fig. 2). For the single frequency case, Goodridge et al. [30] showed experimentally that the ejection acceleration threshold satisfies:

$$
\gamma_{d} \simeq 1.306 \frac{\nu^{1 / 2}(2 \pi m f)^{3 / 2}}{g} .
$$

This scaling law can again be inferred from a balance between the power dissipated by viscosity and the power transferred to the waves. The viscous power per unit area still scales as $P_{\nu} \sim \rho \nu\left(h m f k_{m}\right)^{2} k_{m}^{-1}$. However, the release of droplets means that the vertical velocity of the interface should now also be of the same order of magnitude as the speed $\gamma g /(m f)$ of the forcing vibration. Consequently, the power communicated by the forcing now scales as $[\gamma g /(m f)] \cdot(\rho h) \cdot(\gamma g)$, so balancing both powers yields $\gamma_{d} \sim \sqrt{\nu(m f)^{3} h k_{m}} / g$. The scaling of Eq. (4) then results from the additional constraint that the wave elevation $h$ should be of the order of the wavelength $k_{m}^{-1}$ for the wave to break-up. Equation (4) gives $\gamma_{4 d}=4.81$ and $\gamma_{5 d}=6.71$, again in close agreement with our measurements. This scaling is supposed to be valid as long as $P_{\nu}$ is significantly larger than the power transferred to the liquid/air interface (surface energy). This latter, when expressed per unit of horizontal area, scales as $P_{\sigma} \sim \sigma h^{2} k_{m}^{2} m f$. The power ratio then scales as $P_{\nu} / P_{\sigma} \sim \rho \nu m f /\left(\sigma k_{m}\right) \sim \nu(\rho / \sigma)^{2 / 3}(m f)^{1 / 3}$ for capillary-dominated waves. Goodridge showed experimentally that the transition from viscosity-dominated to surface-tension-dominated instability occurs when

$$
\frac{2 \pi m f \nu^{3} \rho^{2}}{\sigma^{2}} \sim 10^{-5} \Rightarrow \frac{P_{\nu}}{P_{\sigma}} \sim 10^{-2}
$$

Equation 4 is therefore valid as long as $m f \gg$ $10^{-5} \sigma^{2} /\left(2 \pi \nu^{3} \rho^{2}\right) \simeq 0.1 \mathrm{~Hz}$, which is always the case in these experiments.

The droplet diameter and ejection rate have been measured from side-view images captured with high-speed camera (Table I). Only droplets ejected within a horizontal region of diameter $15 \mathrm{~mm}$ are considered (the distance to the camera is here about ten times larger). Close to the ejection threshold, all the droplets have approximately the same size. At larger accelerations, the ejection rate is more important and the droplet size distribution is bimodal: the break-up of each liquid jet results in both one large droplet at the top and some smaller satellite droplets below. By contrast (and contrary to intuition), the two-frequency forcing does not necessarily yield a bimodal distribution, but rather a monodisperse distribution at an intermediate size.

\begin{tabular}{|c|c|c|c|c|}
\hline $\mathrm{f}(\mathrm{Hz})$ & $\left(\gamma_{4}, \gamma_{5}\right)$ & $\mathrm{n}$ & $d_{m}(\mathrm{~mm})$ & $d_{s}(\mathrm{~mm})$ \\
\hline 80 & $(0,7.5)$ & $15 \pm 2$ & 1.48 & 0.06 \\
\hline 80 & $(0,8.0)$ & $30 \pm 5$ & 1.54 & 0.09 \\
& & & 0.64 & 0.09 \\
\hline 80 & $(0,8.5)$ & $40 \pm 5$ & 1.55 & 0.11 \\
& & & 0.72 & 0.19 \\
\hline 64 & $(5.5,0)$ & $15 \pm 2$ & 1.59 & 0.078 \\
\hline 64 & $(5.75,0)$ & $25 \pm 5$ & 1.64 & 0.175 \\
\hline 64 & $(6.0,0)$ & $35 \pm 5$ & 1.63 & 0.168 \\
\hline $80-64$ & $(3.0,4.59)$ & $10 \pm 2$ & 1.72 & 0.058 \\
\hline $80-64$ & $(3.2,4.59)$ & $15 \pm 2$ & 1.79 & 0.068 \\
\hline $80-64$ & $(3.2,4.66)$ & $25 \pm 5$ & 1.75 & 0.102 \\
\hline
\end{tabular}

TABLE I. Ejected droplets for different forcing conditions: number $n$ of droplets from a bath surface of $180 \mathrm{~mm}^{2}$ every $10 \mathrm{~s}$, mean $d_{m}$ and standard deviation $d_{s}$ of the droplet diameter.

\section{DROPLET BOUNCING DYNAMICS}

Without any forcing, an impacting droplet would bounce a few times, less and less high, before it merges with the underlying bath. Sustained rebounds are observed when the forcing acceleration is sufficiently high, typically at least $1 g[3,4,19,20]$. Then the droplet is at all time separated from the bath by an air layer, which is squeezed at each impact and replenished at each take-off (Fig. 5a-b).

The vertical dynamics of sustained bouncing is investigated hereafter for accelerations below the Faraday threshold $\Gamma_{F}: \gamma_{4}$ and $\gamma_{5}$ are increased from 0 to 2.7 and from 0 to 4 respectively, with steps of 0.2 . The absolute vertical position $z_{d}$ of the droplet is extracted by tracking the droplet with image processing. Spatiotemporal diagrams $z_{d}(t)$ are constructed to help identifying various dynamics (e.g. Fig. 5c). They are made by juxtaposing several versions at successive times of the same column of 


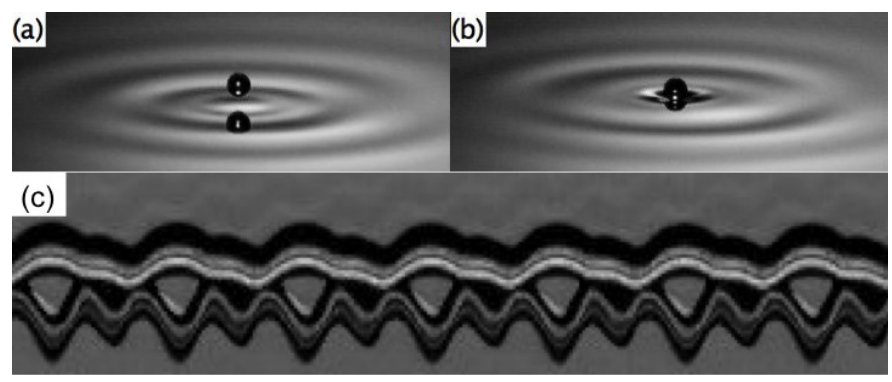

FIG. 5. (a-b) Droplet bouncing on a bath vibrated at $80 \mathrm{~Hz}$ and 2.2 g. (c) Spatio-temporal montage, made by juxtaposing successive vertical slices (here passing through the droplet center). The period of the bouncing sequence is $12.5 \mathrm{~ms}$ (equiv. $80 \mathrm{~Hz}$ ). The top line represents the droplet elevation $z_{d}$, while the bottom one represents its reflection on the bath.

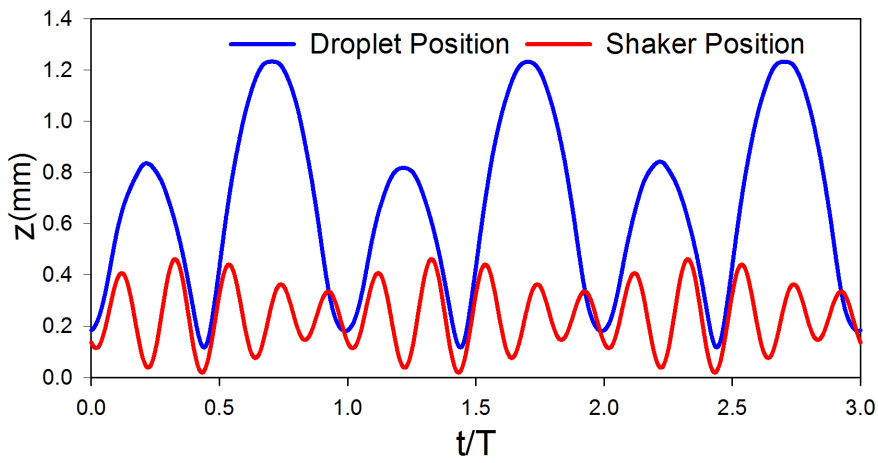

FIG. 6. Evolution of the absolute vertical position of the droplet $z_{d}$ (blue) and the shaker $z_{c}$ (red), for forcing accelerations $\left(\gamma_{4}, \gamma_{5}\right)=(1.5,3.5)$. The first is obtained by image tracking, while the second is reconstructed from accelerometer data. Time is here normalized with the fundamental period $T=1 / f$.

pixels that passes through the center of the droplet. The horizontal dimension here represents time (one pixel per frame). In figure $5 \mathrm{c}$, the droplet motion consists in the alternation of two different rebounds of duration $16.3 \mathrm{~ms}$ and $8.7 \mathrm{~ms}$, while the forcing signal is a pure sinusoid of period $12.5 \mathrm{~ms}$. In figure 6 , the droplet position $z_{d}$ is superimposed to the shaker signal $z_{c}$, which here contains both fourth and fifth harmonics. The droplet performs a bouncing sequence made of two different rebounds, of duration $0.55 T \simeq 34 \mathrm{~ms}$ and $0.45 T \simeq 28.5 \mathrm{~ms}$, which repeats at the same frequency $f$ as the shaking signal.

A wide variety of bouncing patterns is observed as $\gamma_{4}$ and $\gamma_{5}$ are varied (Fig. 7). They are here differentiated according to (i) the period of the pattern and (ii) the number of rebounds $N_{b}$ during $2 T$. When only one harmonic $m$ is present, the pattern period can be $T / m$ (Fig. 7a) or $2 T / m$ (Fig. 7b). When both fourth and fifth harmonics are present, the pattern period is usually $T$ (Fig. 7c-e) or $2 T$ (Fig. 7f), although complex sequences of longer period $3 T$ have been observed for specific ac-

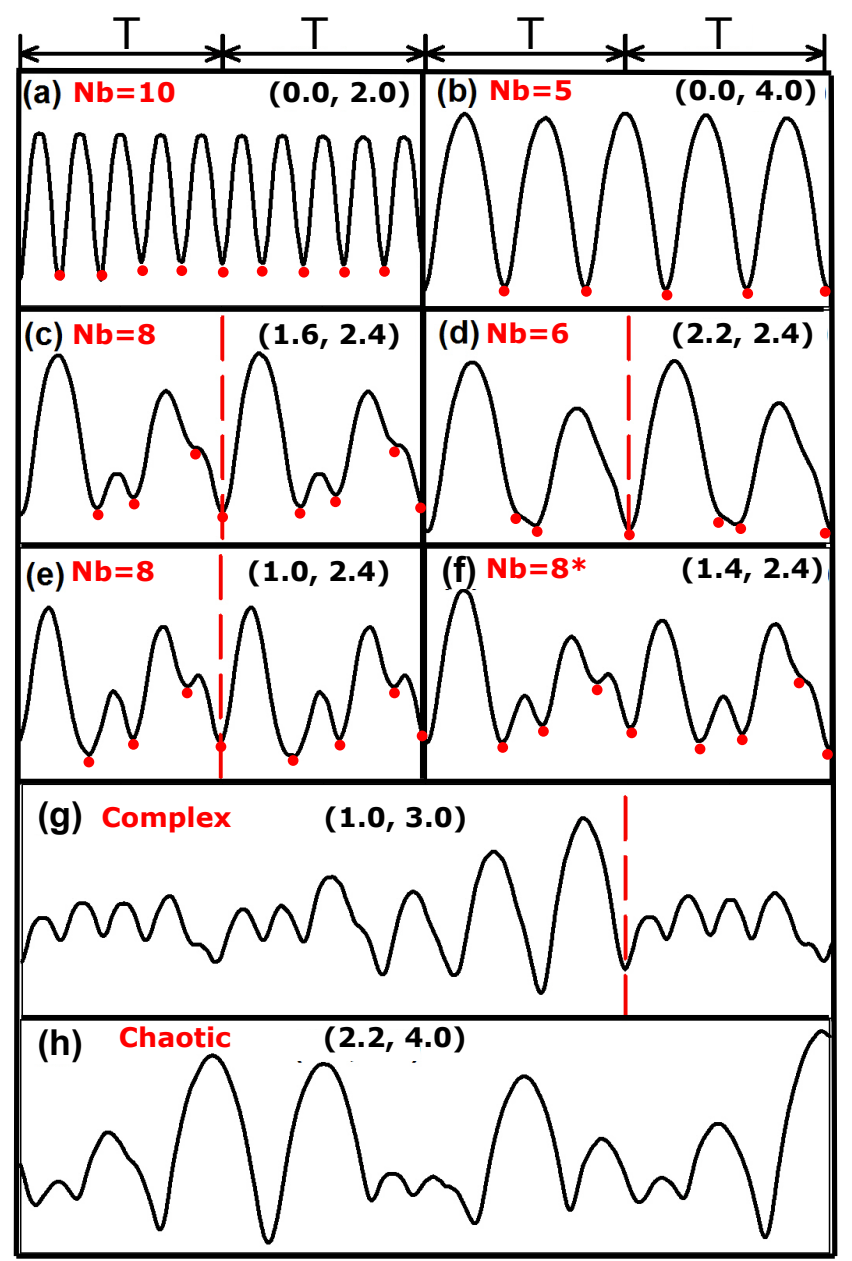

FIG. 7. Various bouncing patterns of a droplet on a bath vibrated with different harmonic amplitudes $\gamma_{m}$ [Eq. 1]. The droplet position (in the lab frame) is measured by image processing (black solid lines). Red dashed lines indicate the periodicity of each pattern. Rebound positions are indicated with red dots. Their number $N_{b}$ during $2 T$ is indicated in red.

celerations around $\left(\gamma_{4}, \gamma_{5}\right) \sim(1,3)$ [Fig. $7 \mathrm{~g}$ ]. Chaotic patterns with no apparent periodicity have also been observed when both accelerations are large, right below the Faraday threshold (Fig. 7h). The number of rebounds $N_{b}$ in two periods also takes different values between 4 and 10. Some patterns can have the same $N_{b}$, although with a different period (Fig. 7e-f).

Bouncing patterns are ordered in distinct zones of the phase diagram $\left(\gamma_{4}, \gamma_{5}\right)$ [Fig. 8]. The number of rebounds $N_{b}$ during $2 T$ decreases when either $\gamma_{m}$ is increased. The pattern period is $T$ in most of the diagram, except at large $\gamma_{5}$ where it is predominantly $2 T$. Period doubling is a common feature of bouncing dynamics that happens as the forcing amplitude is increased [33]. Here, it is only visible for the odd harmonic $m=5$ which halved frequency is not a multiple of $f$ anymore. The complex mode of period $3 T$ and the chaotic pattern both appear in small regions where both harmonics are finely balanced. 


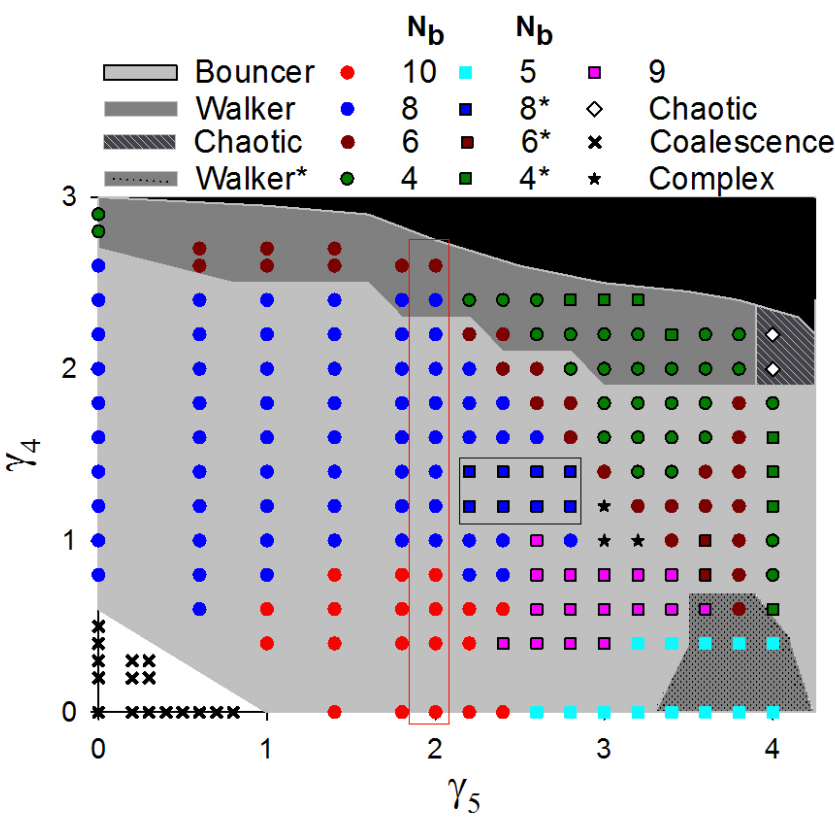

FIG. 8. Phase diagram of bouncing patterns, differentiated by periodicity ( $\circ$ for $T, \square$ for $2 T$, $\star$ for larger periods, $\diamond$ for chaotic patterns) and by number of impacts $N_{b}$ during $2 T$ (colors online). Coalescing droplets (acceleration below the sustained-bouncing threshold) are represented with $\times$. The shading provides information about the horizontal dynamics: (light grey) purely vertical bouncing, (medium grey) regular walking at $64 \mathrm{~Hz}$, (medium grey, dotted) regular walking at $80 \mathrm{~Hz}$, (dark grey) chaotic walking. The Faraday instability occurs in the black region, at the top right of the diagram.

Bifurcations between different bouncing patterns can be analyzed through the evolution of a bouncing sequence with increasing $\gamma_{4}$ (and fixed $\gamma_{5}$ ), as shown in Figure 9. The pattern period is always equal to $T$ (except at $\gamma_{4}=$ 0 where it is $T / 5)$, while the number of rebounds $N_{b}$ decreases from 10 to 6 . The duration of the first flight (on the left) increases with increasing $\gamma_{4}$. As a result, the second flight shortens and disappears in $\gamma_{4}=1.2$ through a first grazing bifurcation $[16,34]$ that brings $N_{b}$ to 8: the droplet misses the impact before the second flight, as the downward speed of the bath becomes greater than the droplet impacting speed. When the first flight finally ends, the droplet impacts but does not take off immediately, it sticks to the bath until the downward acceleration of this latter is sufficient for taking off. The first and third flights finally merge in $\gamma_{4}=2.6$, through a second grazing bifurcation that brings $N_{b}$ to 6 . The duration of flights 4 and 5 does vary with increasing $\gamma_{4}$, but it does not lead to any change of topology. Another kind of bifurcation, commonly called period doubling, is seen from Fig. 7e to $7 f$ [16].

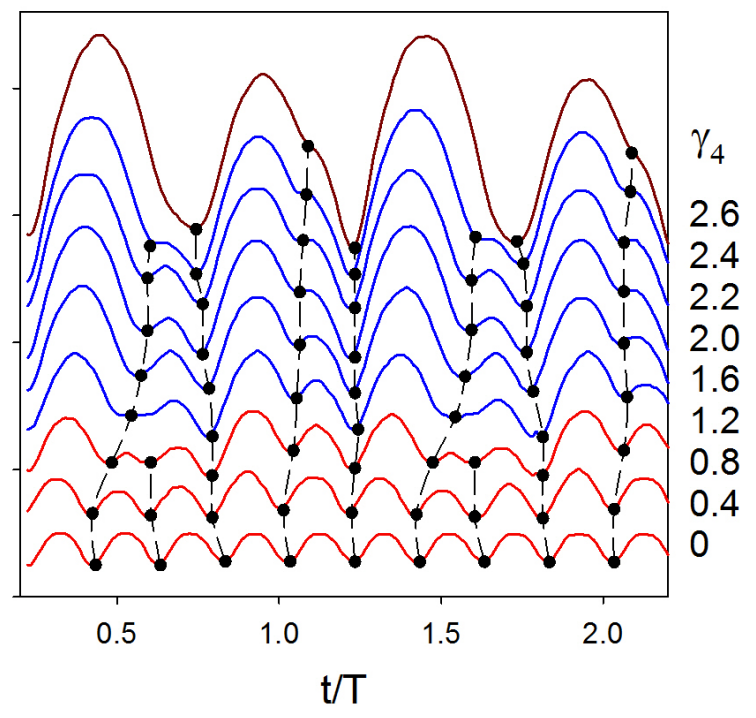

FIG. 9. Evolution of bouncing patterns as $\gamma_{4}$ is increased from 0 to 2.6 , for fixed $\gamma_{5}=2$. The position is measured by image processing (colored solid lines online, according to color scheme of Fig. 8). Black dots (connected with dashed lines) represent droplet impacts where the droplet velocity is reversed. A grazing bifurcation occurs at the upper end of each dashed line.

\section{VERTICAL CHAOS AND HORIZONTAL DYNAMICS}

In the chaotic regime at $\left(\gamma_{4}, \gamma_{5}\right)=(2.2,4)$, the bouncing sequence showed no periodicity for more than $2.5 \mathrm{~s}$, i.e. $40 T$ (Fig. 10, top). The corresponding histogram of flight times (compiled from the recording of 30 trajectories, each of duration $5 \mathrm{~s}$, recorded at $800 \mathrm{fps}$ ) comprises distinct peaks at $T / 5=12.5 \mathrm{~ms}$ and $T / 2.5=$ $25 \mathrm{~ms}$, which are reminiscent of fifth-harmonic timescales $\left(\gamma_{4}=0\right)$. By contrast, throughs are observed around $T / 4 \simeq 15.6 \mathrm{~ms}$ and $T / 2 \simeq 31.2 \mathrm{~ms}$ (in correspondence with the timescales of the fourth harmonic). The peak at $34 \mathrm{~ms}$ does not evoke any immediate timescale of the forcing signal. Flight times higher than $36 \mathrm{~ms}$ are rare, owing to the finite energy provided by the forcing and the increase of viscous dissipation with impact speed (and flight time) [19, 20].

In most of the phase diagram (Fig. 8), the droplet trajectory is purely vertical; it is called a bouncer. The spatio-temporal diagram of Fig. 11a confirms that in such condition the droplet does not move horizontally. At single frequency, droplets can also walk, i.e. experience sustained horizontal motion, when the shaking amplitude is lower than but close to the Faraday threshold [10]. The spatio-temporal of Fig. 11b reveals that in such case the velocity is constant. Regular walking also exists when a second forcing frequency is present, as confirmed in Figure 8 . 


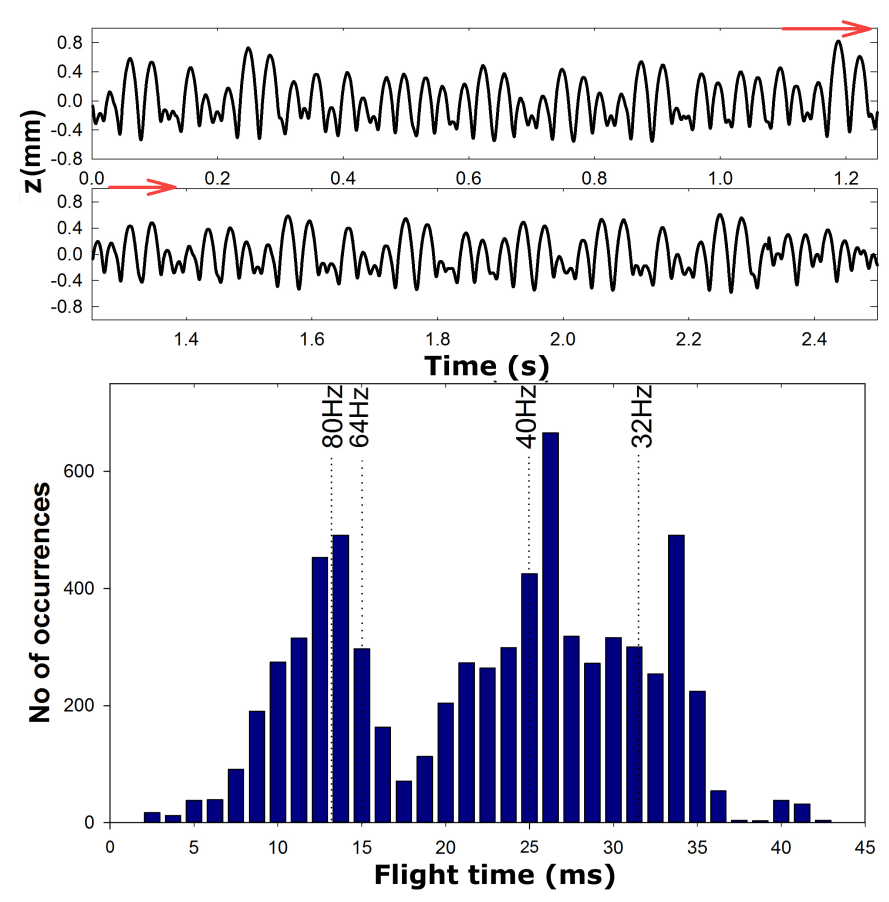

FIG. 10. Chaotic bouncing pattern recorded for $2.5 \mathrm{~s}$ at $\left(\gamma_{4}, \gamma_{5}\right)=(2.2,4)$. (Top): Evolution of the droplet vertical position, recorded for $2.5 \mathrm{~s}$. (Bottom) Histogram of flight times.

When the vertical trajectory is chaotic, so is the horizontal motion (Fig. 11c): the trajectory is jerky, the droplet alternates periods of walk and rest (purely vertical bouncing, and almost vertical segments in Fig. 11c). Often, after a rest period, the walk continues in its original direction (Fig. 11c, d). Nevertheless, rest periods are sometimes the occasion to change orientation, as seen in Fig. $11 \mathrm{~h}$ where the droplet randomly explores the whole bath surface. The snapshots of Figure 11e-g show that the shape of the wave-field is different during walking and rest phases.

The typical evolution of the instant velocity $V$ with travelled distance $d$ is shown in Figure 12a, where $d$ is the time integral of $V$. Velocity $V(d)$ oscillates between 0 (rest) and a maximum of about $10 \mathrm{~mm} / \mathrm{s}$, which is approximately the walking velocity $V_{s} \simeq 10.5 \mathrm{~mm} / \mathrm{s}$ at single frequency. Consequently the average velocity $V_{m} \simeq 4.82 \mathrm{~mm} / \mathrm{s}$ is smaller than $V_{s}$. The histogram of Figure $12 \mathrm{~b}$ indicates that speeds in the range $\left[0, V_{s}\right]$ are approximately equiprobable. The oscillations of $V(d)$ reveal a characteristic distance of about 4 to $5 \mathrm{~mm}$ that separates the droplet positions at two successive periods of rest (Fig. 12a). This distance appears as a peak in the power spectrum of $V(d)$ (Fig. 12c). A zoom on this spectrum around $d \simeq 5 \mathrm{~mm}$ reveals three maxima at $d \simeq 4.82 \mathrm{~mm}, d \simeq 5.28 \mathrm{~mm}$ and $d \simeq 5.60 \mathrm{~mm}($ Fig. $12 \mathrm{~d})$, which are very close to the Faraday wavelengths $\lambda_{4}$ and $\lambda_{5}$ associated to both harmonics. These observations suggest that the waves strongly shape the horizontal trajec-
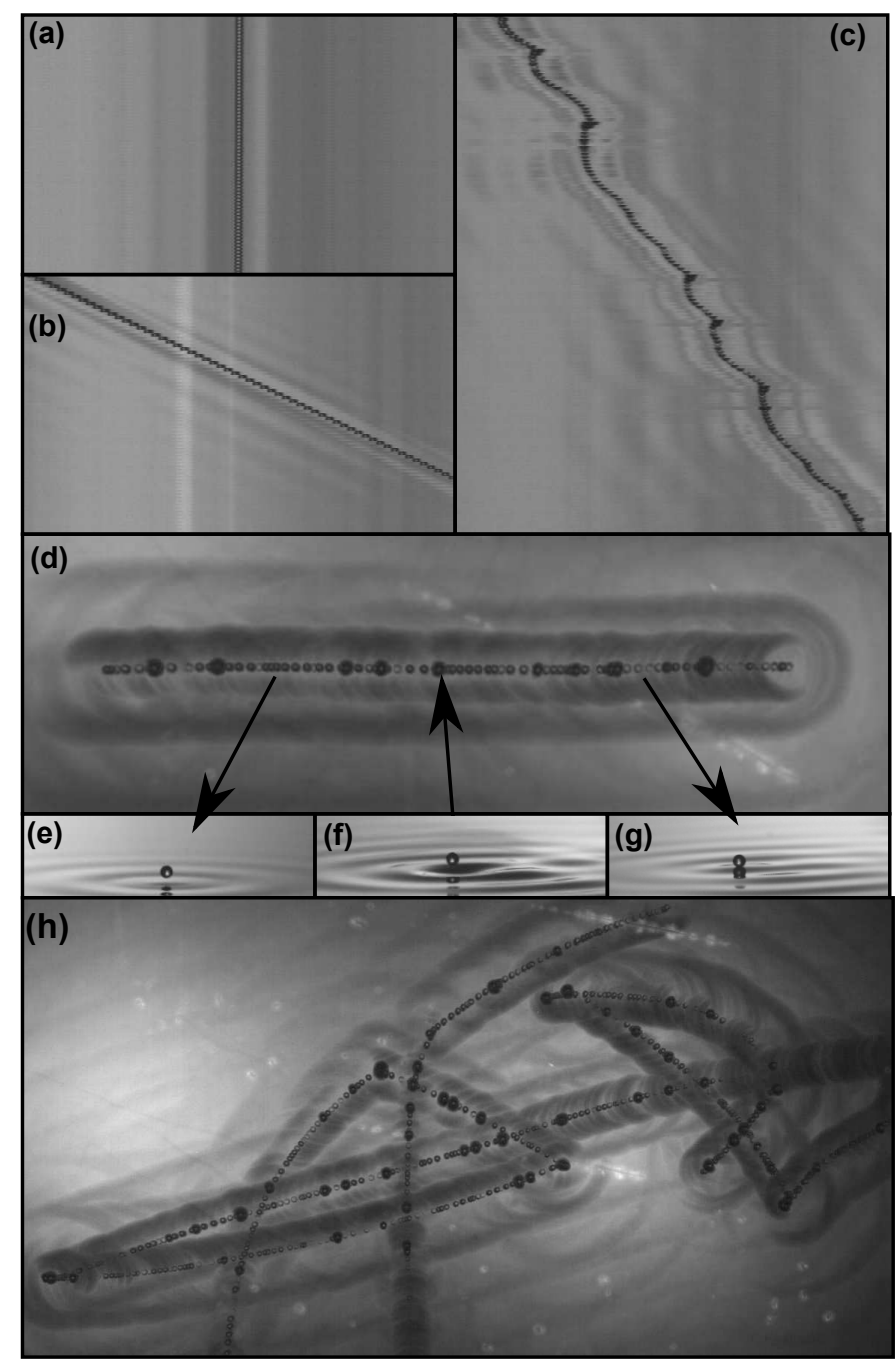

FIG. 11. Horizontal dynamics. (a-c) Spatio-temporal diagrams of the droplet horizontal motion (vertical axis $=$ time). (a) Purely vertical bouncing at $\left(\gamma_{4}, \gamma_{5}\right)=(2.2,0.8)$. (b) Regular walking at velocity $10.5 \mathrm{~mm} / \mathrm{s}$ at $\left(\gamma_{4}, \gamma_{5}\right)=(2.8,0.2)$. (c-h) Chaotic walking at average velocity $4.82 \mathrm{~mm} / \mathrm{s}$ at $\left(\gamma_{4}, \gamma_{5}\right)=(2.2,4) . \quad(\mathrm{d}, \mathrm{h})$ Top-views of the chaotic walker trajectory, obtained by superimposing frames taken at successive time. (e-g) Side-view snapshots of the same chaotic walker (and associated wave-field) at different moments of its jerky motion.

tory of the droplet. When the droplet starts from rest, it builds up a wave field that is locally unstable (as shown in [35] for the single frequency case). It then starts walking, but the coherent horseshoe wave pattern characteristic of regular walkers [9] does not form here, owing to the complex wave interference of contributions from irregular impacts. After having travelled a distance of the order of one Faraday wavelength, the droplet lands at a position where the wave slope is relatively small, so it slows down and almost stops walking. It then rebuilds the wave field and starts walking again, in a possibly different direction.

Oscillations of the horizontal velocity at the scale of 

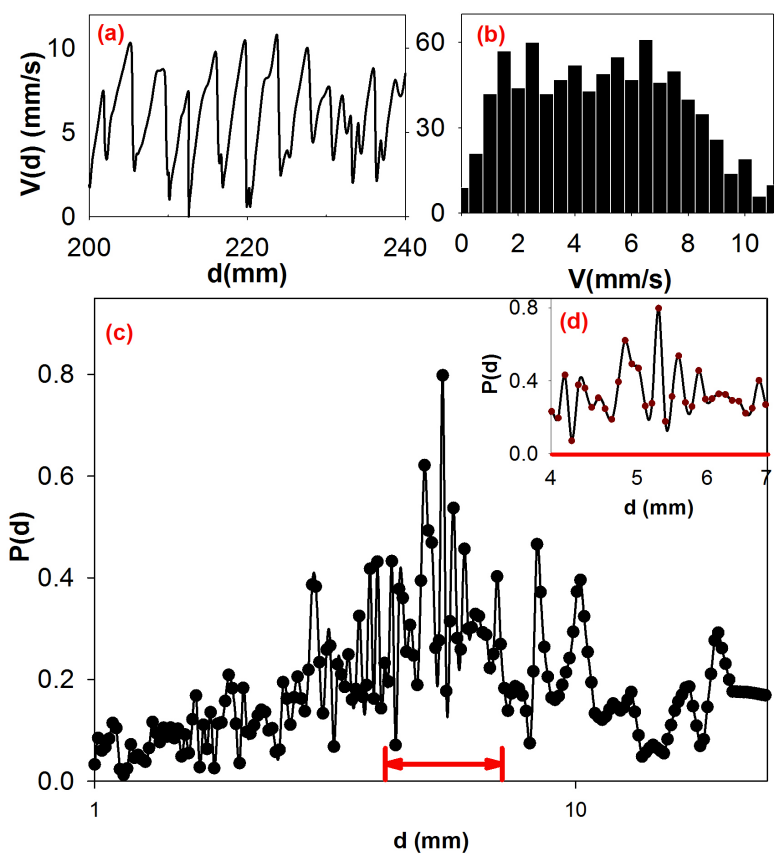

FIG. 12. Instant velocity $V$ at $\left(\gamma_{4}, \gamma_{5}\right)=(2.2,4)$ : (a) as a function of distance travelled $d$, (b) histogram for several trajectories, (c) power spectrum $|P(d)|$, and (d) zoom on the central peak of the power spectrum.

the Faraday wavelength have already been observed for walkers in several configurations $[13,14,36]$. In particular, we showed in a recent work that for walkers confined in cavities, the Faraday wavelength is the elementary step of a $2 \mathrm{D}$ random walk $[37,38]$ which statistics is shaped by the wave in accordance with Schrödinger equation. In the present work, the walker also explores the bath surface with steps of approximate size $\lambda_{4-5}$ (Fig. 11h). Figure 13 represents the average distance $D(T)$ travelled by the walker in a duration $T$. It is defined as

$$
D(T)=\sqrt{\langle\|\mathbf{x}(t+T)-\mathbf{x}(t)\|\rangle}
$$

where $\mathbf{x}(t)$ is the horizontal position of the droplet at time $t$. The average $\langle\cdot\rangle$ includes both a time average and an ensemble average over 52 trajectories. The distance $D$ increases almost linearly with time $T$ so the motion is ballistic (of average speed $5.7 \mathrm{~mm} / \mathrm{s}$ ), at least on timescales of a few seconds [38]. Indeed, the walker does not change direction at every step (e.g. in Fig. 11d and 11h). The average angular velocity of the droplet is around $0.08 \mathrm{rad} / \mathrm{s}$, so any diffusion-like behavior could only be observed at a timescale larger than $2 \pi / 0.08 \simeq 79 \mathrm{~s}$ [38]. Unfortunately, our recording time was limited to $55 \mathrm{~s}$ so the diffusion behavior could not be evidenced.

In previous works on walkers, the chaos in the horizontal trajectory always resulted from the confinement of the particle and associated wave. The confinement was ensured by a central force [14, 39], by a Coriolis force

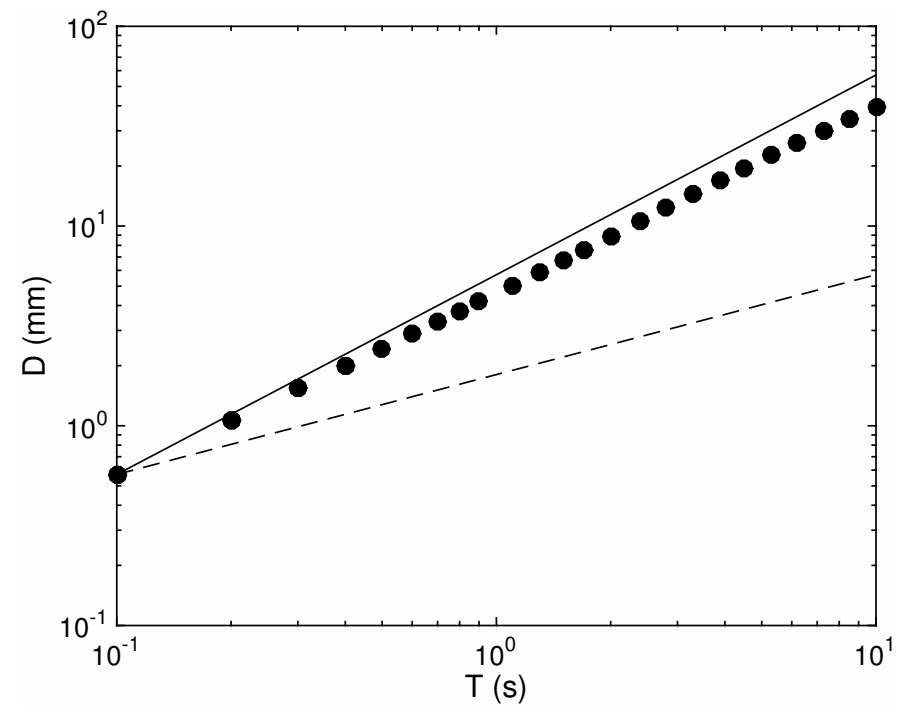

FIG. 13. Average distance $D(T)$ travelled in a given time $T$. The statistics is made on 52 different trajectories, all taken in the chaotic regime $\left(\gamma_{4}, \gamma_{5}\right)=(2.2,4)$. The solid line represents the ballistic law $D=5.7 T$, and the dashed line represents the diffusive law $D=1.8 \sqrt{T}$.

$[12,40]$, or by physical boundaries $[13,38]$. In each case, the vertical trajectory could be assumed periodic. The aperiodic horizontal motion would only result from the chaotic droplet interaction with the waves that it previously left in its wake. By contrast, horizontal chaos is obtained in the absence of confinement in this work. Instead, it directly originates from the chaos in the vertical dynamics.

\section{CONCLUSION}

We have here explored experimentally the dynamics of a bath vertically vibrated with a signal composed of two harmonics. The Faraday threshold (spontaneous appearance of standing surface waves) and the Goodridge threshold (ejection of droplets from the break-up of Faraday waves) have been determined as functions of the forcing amplitudes. Droplets can bounce periodically below the Faraday threshold. Varying each forcing amplitude revealed a myriad of bouncing modes. Among them, regular walkers (with constant non-zero horizontal velocity) are observed when the amplitude of one harmonic is near its Faraday threshold. The bouncing dynamics becomes chaotic below the Faraday threshold, when both harmonic amplitudes are important. In this case, the horizontal motion becomes an irregular succession of walking and stopping, which characteristic size is given by the excited Faraday wavelengths. In several explored configurations did confined individual walkers exhibit chaotic behaviors as soon as the Faraday threshold was approached. Although the vertical motion was periodic, the walker had the possibility to re-explore re- 
gions where it had already left waves. Horizontal chaos here originates from a different mechanism: the irregular vertical bouncing dynamics forced simultaneously at two frequencies. The classical interaction of particles and waves is incredibly rich and insightful [5]. The inclusion of a second forcing frequency, first reported in this work, yields a series of specific periodic and chaotic behaviors, which certainly require further investigation.
Acknowledgements This research was performed in the framework of the Quandrops project, financially supported by the Actions de Recherches Concertees (ARC) of the Belgium Wallonia-Brussels Federation under contract No. 12-17/02. This research has also been funded by the Interuniversity Attraction Poles Programme (IAP 7/38 MicroMAST) initiated by the Belgian Science Policy Office. The authors thank J.W.M. Bush, D. Harris, Y. Couder, M. Hubert and P. Schlagheck for fruitful discussions.
[1] O. Reynolds, Chem. News 44, 211 (1881).

[2] O. W. Jayaratne and B. J. Mason, Proc. R. Soc. London A 280, 545 (1964).

[3] Y. Couder, E. Fort, C.-H. Gautier, and A. Boudaoud, Phys. Rev. Lett. 94, 177801 (2005).

[4] T. Gilet, D. Terwagne, N. Vandewalle, and S. Dorbolo, Phys. Rev. Lett. 100, 167802 (2008).

[5] J. W. M. Bush, Annu. Rev. Fluid Mech. 47, 269 (2015).

[6] J. W. M. Bush, Physics Today 68, 47 (2015).

[7] T. B. Benjamin and F. Ursell, Proc. R. Soc. London 225, 505 (1954).

[8] Y. Couder, S. Protiere, E. Fort, and A. Boudaoud, Nature 437, 208 (2005).

[9] A. Eddi, E. Sultan, J. Moukhtar, E. Fort, M. Rossi, and Y. Couder, J. Fluid Mech. 674, 433 (2011).

[10] Y. Couder and E. Fort, Phys. Rev. Lett. 97, 154101 (2006).

[11] A. Eddi, D. Terwagne, E. Fort, and Y. Couder, Europhys. Lett. 82, 44001 (2008).

[12] E. Fort, A. Eddi, A. Boudaoud, J. Moukhtar, and Y. Couder, PNAS 107, 17515 (2010).

[13] D. M. Harris, J. Moukhtar, E. Fort, Y. Couder, and J. W. M. Bush, Phys. Rev. E 88, 011001 (R) (2013).

[14] S. Perrard, M. Labousse, M. Miskin, E. Fort, and Y. Couder, Nat. Comm. 5, 3219 (2014).

[15] T. Gilet and J. W. M. Bush, Phys. Rev. Lett. 102, 014501 (2009).

[16] T. Gilet, N. Vandewalle, and S. Dorbolo, Phys. Rev. E 79, 055201 (R) (2009).

[17] S. Protiere, A. Boudaoud, and Y. Couder, J. Fluid Mech. 554, 85 (2006).

[18] S. Dorbolo, D. Terwagne, N. Vandewalle, and T. Gilet, New J. Phys. 10, 113021 (2008).

[19] T. Gilet and J. W. M. Bush, J. Fluid Mech. 625, 167 (2009).

[20] J. Molacek and J. W. M. Bush, J. Fluid Mech. 727, 582 (2013).

[21] D. Terwagne, F. Ludewig, N. Vandewalle, and S. Dorbolo, Phys. Fluids 25, 122101 (2013).

[22] D. M. Harris, T. Liu, and J. W. M. Bush, Exp. Fluids 56, 83 (2015).

[23] S. Protiere, Gouttes rebondissantes : une association onde-particule a echelle macroscopique, Ph.D. thesis, Universite Paris VII (2007).

[24] J. Molacek and J. W. M. Bush, J. Fluid Mech. 727, 612 (2013).

[25] M. Hubert, D. Robert, H. Caps, S. Dorbolo, and N. Vandewalle, Phys. Rev. E 91, 023017 (2015).
[26] J. Bechhoefer, V. Ego, S. Manneville, and B. Johnson, J. Fluid Mech. 288, 325 (1995).

[27] W. S. Edwards and S. Fauve, Phys. Fluids A 5, S10 (1993).

[28] T. Besson and W. S. Edwards, Phys. Rev. E 54, 507 (1996).

[29] C. L. Goodridge, W. T. Shi, and D. P. Lathrop, Phys. Rev. Lett. 76, 1824 (1996).

[30] C. L. Goodridge, W. Tao Shi, H. G. E. Hentschel, and D. P. Lathrop, Phys. Rev. E 56, 472 (1997).

[31] C. L. Goodridge and H. G. E. Hentschel, Phys. Rev. Lett. 82, 3062 (1999).

[32] S. C. Tsai, S. K. Lin, R. W. Mao, and C. S. Tsai, Phys. Rev. Lett. 108, 154501 (2012).

[33] J. M. Luck and A. Mehta, Phys. Rev. E 48, 3988 (1993).

[34] M. di Bernardo, C. J. Budd, A. R. Champneys, and P. Kowalczyk, Piecewise-smooth dynamical systems: Theory and applications, edited by S. S. Antman, J. E. Marsden, and L. Sirovich, Applied Mathematical Sciences No. 163 (Springer, London, 2008).

[35] A. U. Oza, R. R. Rosales, and J. W. M. Bush, J. Fluid Mech. 737, 552 (2013).

[36] A. U. Oza, D. M. Harris, R. R. Rosales, and J. W. M. Bush, J. Fluid Mech. 744, 404 (2014).

[37] T. Gilet, Phys. Rev. E 90, 052917 (2014).

[38] T. Gilet, Phys. Rev. E 93, 042202 (2016).

[39] S. Perrard, M. Labousse, E. Fort, and Y. Couder, Phys. Rev. Lett. 113, 104101 (2014).

[40] D. M. Harris and J. W. M. Bush, J. Fluid Mech. 739, 444 (2014).

\section{Appendix A: Scaling law of the Faraday threshold}

In this appendix, we provide a short derivation of the scaling law for the Faraday threshold in the low-viscosity limit.

Benjamin and Ursell [7] showed that for an inviscid liquid vibrated at an angular frequency $\omega_{0}$ and acceleration $\gamma g$, the amplitude $a$ of subharmonic Faraday waves satisfies the Matthieu equation

$$
\frac{d^{2} a}{d T^{2}}+\left[\frac{1}{4}-2 \epsilon \cos T\right] a=0
$$

where $T=\omega_{0} t$ is the dimensionless time, $\epsilon=\gamma g k /\left(2 \omega_{0}^{2}\right)$ is the dimensionless forcing, and $k$ is the wave number 
obtained from the dispersion relation

$$
\frac{\omega_{0}^{2}}{4}=g k+\frac{\sigma}{\rho} k^{3}
$$

The presence of a finite (but low) viscosity can be taken into account by adding a phenomenological damping term to the Matthieu equation:

$$
\frac{d^{2} a}{d T^{2}}+2 \alpha \frac{\nu k^{2}}{\omega_{0}} \frac{d a}{d T}+\left[\frac{1}{4}-2 \epsilon \cos T\right] a=0
$$

where the dimensionless coefficient $\alpha$ is here kept as a fitting parameter. The resulting damped amplitude can be written:

$$
a(T)=y(T) e^{-\Gamma T}, \quad \Gamma=\frac{\alpha \nu k^{2}}{\omega_{0}},
$$

Then, in the low-viscosity limit $(\Gamma \ll 1 / 4), y(T)$ satisfies again the Matthieu equation:

$$
\frac{d^{2} y}{d T^{2}}+\left[\frac{1}{4}-2 \epsilon \cos T\right] y=0
$$

If the damping time is much larger than the forcing period, a multiple-scale expansion can be performed:

$$
y=y_{0}(T, \tau)+\epsilon y_{1}(T, \tau)
$$

where $T$ and $\tau=\epsilon T$ are the fast and slow scales respectively. The zeroth-order solution is obtained by solving

$$
\frac{\partial^{2} y_{0}}{\partial T^{2}}+\frac{y_{0}}{4}=0 \Rightarrow y_{0}=A(\tau) e^{i T / 2}+A^{*}(\tau) e^{-i T / 2}
$$

Then the first order solution satisfies:

$$
\frac{\partial^{2} y_{1}}{\partial T^{2}}+\frac{y_{1}}{4}=2 y_{0} \cos T-2 \frac{\partial^{2} y_{0}}{\partial T \partial \tau}
$$

Cancelling secular terms $e^{i T / 2}$ and $e^{-i T / 2}$ in this latter equation yields $A(\tau)=A_{0} e^{\tau}$. So to the first order, the amplitude $a$ of the waves evolves as:

$$
a(T) \simeq A_{0} e^{(\epsilon-\Gamma) T} \cos (T / 2)
$$

The Faraday threshold then corresponds to $\epsilon=\Gamma$, which yields

$$
\gamma=\gamma_{F}=2 \alpha \frac{\nu k \omega_{0}}{g}
$$

